\title{
The risk of lymph node metastases and their impact on survival in patients with appendiceal neuroendocrine neoplasms: a systematic review and meta-analysis of adult and paediatric patients
}

\author{
Kosmas Daskalakis $\mathbb{D}^{1,2} \cdot \mathrm{Krystallenia} \mathrm{Alexandraki}^{2} \cdot$ Evanthia Kassi $^{2,3} \cdot$ Marina Tsoli $^{2} \cdot$ Anna Angelousi $^{2}$. \\ Athanasia Ragkousi ${ }^{2} \cdot$ Gregory Kaltsas $^{2,4,5}$
}

Received: 28 May 2019 / Accepted: 21 August 2019 / Published online: 6 September 2019

(c) The Author(s) 2019

\begin{abstract}
Background There are no clear histopathological parameters determining the risk of lymph node (LN) metastases and appropriateness of completion prophylactic right hemicolectomy (RHC) in patients with appendiceal neuroendocrine neoplasms (ANENs).

Materials and methods The PubMed, Cochrane Library, Embase, Web of Science and SCOPUS databases were searched up to November 2018. Quality/risk of bias was assessed using the Newcastle-Ottawa Scale (NOS).

Results A total of 526 articles were screened. In 11 adult and 3 paediatric studies, 602 and 77 unique patients, respectively, with ANEN and undergoing RHC, were included. The rate of LN metastases for a cutoff size >10 mm was $48.6 \%$ (vs $12.1 \%$ for lesions $<10 \mathrm{~mm}$ ) among adult patients, with an odds ratio (OR) of 4.8 (95\% CI, 1.5-15.8). For $20 \mathrm{~mm}$ size cutoff, these figures were $61 \%$ (vs $28.2 \%$ for lesions $<20 \mathrm{~mm}$ ) with an OR of 3.2 (95\% CI, 1.3-7.8). Vascular-, lymph vessel- and perineural invasions were identified as predictive factors for LN metastases in adult patients. In paediatric patients, there were no strong morphological predictors for LN metastases. The 10-year disease-specific survival (DSS) for adult patients without LN metastases was $99.2 \%$ vs $95.6 \%$ in patients with LN (OR: $0.2 ; 95 \%$ CI, 0.02-2.4). The complication rate of prophylactic RHC was $11.4 \%$.

Conclusions This meta-analysis demonstrates that tumour size $>20 \mathrm{~mm}$ as well as $>10 \mathrm{~mm}$ and/or vascular-, lymph vesseland perineural invasions are associated with increased risk for LN metastases in adult patients with ANEN. The prognostic value of LN positivity remains to be determined in further studies with long-term follow-up.
\end{abstract}

Keywords Appendiceal neuroendocrine neoplasms $\cdot$ Locoregional lymph node metastases $\cdot$ Prophylactic right hemicolectomy

Supplementary information The online version of this article (https:// doi.org/10.1007/s12020-019-02072-y) contains supplementary material, which is available to authorized users.

Kosmas Daskalakis

kosmas.das@gmail.com

1 Department of Surgical Sciences, Uppsala University, Uppsala, Sweden

2 1st Department of Propaupedic Internal Medicine, Endocrine Oncology Unit, Laiko Hospital, National and Kapodistrian University of Athens, Athens, Greece

\section{Introduction}

Appendiceal neuroendocrine neoplasms (ANENs) arise from the subepithelial neuroendocrine cells of the appendiceal wall and are generally discovered incidentally at appendectomy for acute appendicitis [1, 2]. They commonly exhibit a benign clinical course with a minority

3 Department of Biological Chemistry, Medical School, National and Kapodistrian University of Athens, Athens, Greece

4 Clinical Sciences Research Laboratories, Warwick Medical School, University of Warwick, University Hospital, Coventry, UK

5 Centre of Applied Biological \& Exercise Sciences, Faculty of Health \& Life Sciences, Coventry University, Coventry, UK 
developing locoregional lymph node (LN) metastases. Few cases have been reported with distant stage disease [3]. The literature shows great diversity in terms of disease-specific mortality and its association with the presence of locoregional metastatic disease in LNs of the mesentery, as well as the type and extent of surgery undertaken. This diversity is mainly attributed to the fact that in the majority of series, not only well-differentiated (WD) ANENs were included $[4,5]$.

Due to the indolent course of the disease and because there is currently no adjuvant therapy that is known to improve overall survival (OS) in ANENs, surgery is considered the mainstay of treatment. Appendectomy alone has traditionally been considered an adequate treatment for ANENs $<10 \mathrm{~mm}$, whereas a completion prophylactic right hemicolectomy (RHC) following appendectomy is generally advocated for lesions $>20 \mathrm{~mm}$. This approach to ANEN management is also in accordance with current European Neuroendocrine Tumour Society (ENETS) guidelines [6]. However, histopathological parameters determining the risk of LN metastases and their associated mortality in adult and paediatric patients with an ANEN measuring between 10 and $20 \mathrm{~mm}$ have not been clearly defined and are still a matter of debate.

Earlier systematic reviews, including a recent appraisal from ENETS on unmet needs in ANEN management included only retrospective, observational, institutional and registry-based studies, as no randomized trial on ANENs is available to date [7]. Importantly, several large studies on this topic were published during recent years contributing valuable new evidence in the field of ANENs. To date, predominant predictive factors associated with disease outcomes in terms of locoregional metastatic propensity are size and grade of the primary tumour as defined by the Ki67 proliferation index. Some investigators also argue the clinical importance of serosal penetration, i.e. mesoappendix invasion and other histopathological parameters.

Although the various ANEN size cut-offs for completion prophylactic RHC used in recent publications have potentially caused a loss of valuable information, light has been shed on the predictive value of other histopathological factors of the appendectomy specimen with respect to the risk for locoregional $\mathrm{LN}$ metastases. There remains a great need for summarized evidence to address the "grey zones" in ANEN management and in particular appropriateness, effectiveness and safety of completion prophylactic RHC in ANEN cases. Foremost, the risk of LN metastases as a function of tumour size and their morphological characteristics remains to be determined.

Our aim was to compare the rate of LN metastases and their impact on survival for adult and paediatric patients with ANENs undergoing RHC at different size cutoffs, with and without the presence of specific morphological parameters at histopathology, and also to assess the rate of complications following RHC in ANENs.

\section{Patients and methods}

\section{Study selection}

National registry studies, along with retrospective cohort studies on ANEN in adult and paediatric patients undergoing surgery, were eligible for inclusion. The outcomes that were required for eligibility included at least two of the following terms: tumour size, location, grade, mesoappendix invasion, vascular invasion, lymph vessel invasion, perineural invasion and LN metastases, OS and complications. A sample size of at least ten ANEN patients undergoing RHC was necessary for study inclusion, at least in the adult ANEN population. Studies reporting data on gobbler cell appendiceal tumours and tumours of mixed histopathology together with WD ANEN were excluded. Only the latest eligible study was selected among multiple reports from the same research group, institution or national registries, e.g. the SEER database. In cases with overlap in patient cohorts of two studies, the most recent and largest study was included, unless data were available at the individual level, allowing for exclusion of duplicate cases. Evolving classifications in the histopathology of gastroenteropancreatic NENs mainly concern pancreatic NENs and did not affect the selection of eligible studies. We followed Preferred Reporting Items for Systematic Reviews and Meta-Analyses (PRISMA) guidelines for reporting [8]. No study protocol for this meta-analysis was published or registered before the study was undertaken. The primary endpoint was to evaluate the risk for LN metastases using different size cutoffs and other morphological parameters at histopathology in order to select best adult and paediatric candidates for RHC. The secondary endpoints were to evaluate the impact of $\mathrm{LN}$ positivity and that of prophylactic RHC in survival. In particular the inclusion criteria included pathological ANEN diagnosis after surgery, a RHC specimen for histopathological evaluation and the presence of size 10 and $20 \mathrm{~mm}$ cutoffs as well as that of other morphological parameters at histopathology.

\section{Search strategy}

To identify studies and determine eligibility, we conducted a systematic search in the PubMed, Cochrane Library, Embase, Web of Science and SCOPUS databases. Search terms included "appendiceal neuroendocrine tumour", "appendectomy", "surgery", "resection", "RHC" and "hemicolectomy", which were all used in combination with the boolean operators AND and OR. The search terms were 
input as free text. All eligible titles and abstracts were assessed in duplicate by two of the authors (K.D. and K.A.). Full manuscripts were examined as necessary to finalize the study selection. Reference lists of studies reviewed for eligibility were also searched, to identify additional studies.

\section{Data extraction}

Data were independently extracted by two of the authors (K. D. and K.A). The primary outcome was defined as the prevalence of LN metastases at RHC using different morphological parameters at histopathology. We defined the following secondary outcomes: mortality associated with LN metastases and complication rate occurring in ANEN patients undergoing prophylactic RHC. Potentially eligible studies with double zero cells of investigated outcomes were not included in the analysis. The study hypothesis was formulated before data collection. Any discrepancies concerning the extracted data between the two authors were resolved by consensus or by consultation of a third author (G.K.).

\section{Risk for bias}

Our classification of observational institutional and registrybased studies followed classical epidemiologic study designs (e.g. case-control and cohort study), with the key element of this being based on analysis features [9]. For quality assessment of the cohort studies included we applied a score system and assessed the studies in accordance with the Newcastle-Ottawa Scale (NOS) criteria. The total score range was from 0 (worst) to 9 (best) for case-control and cohort studies, with a score of at least 6 suggesting high quality [10].

\section{Statistical analysis and exploration of heterogeneity}

Statistical analyses were completed using STATA 14.0 software (StataCorp, 2015. Stata Statistical Software: Release 14. College Station, TX: StataCorp LP). We adopted a random-effects model to combine the summary statistics and reported pooled OR for primary and secondary outcomes. Statistical heterogeneity was evaluated by the $\mathrm{I}^{2}$ method and the $\chi^{2}$ test to calculate $p$-values; $I^{2}$ values $>50 \%$ suggested increased heterogeneity. Potential publication bias and small study effects were assessed by visually inspecting funnel and Galbraith plots and conducting complementary tests (Egger's and Harbord's tests) as appropriate. We tested the small sample size effect in paediatric ANEN population using Begg test and trim and fill method to correct the ORs if necessary. In addition to principal analyses, random-effects univariate metaregression analyses were performed to account for statistical interstudy heterogeneity as appropriate. The results were given as OR with $95 \% \mathrm{CI}$, and $p$-values, as appropriate. The $5 \%$ level $(p$-value $<0.05)$ was set to indicate statistical significance.

\section{Results}

\section{Characteristics of included studies}

A total of 526 articles were screened. From 11 adult and 3 paediatric studies, 602 and 77 unique patients with ANEN undergoing RHC were included, respectively. The literature search and the selection of included studies are presented in the PRISMA flow diagram (Fig. 1). The characteristics of the included studies are summarized in Table 1, including information on funding and potential conflict of interest.

\section{Quality assessment and risk of bias within studies}

The results of the quality assessment of each study are presented in Table 2 (NOS template). We identified no randomized trials. All studies were observational cohort studies based on retrospective analysis of institutional or registry data. The variety of studies included did not vary considerably. Factors contributing to lower NOS scores were small sample sizes, ambiguity over ANEN inclusion criteria, inadequate follow-up and/or many patients lost to follow-up, lack of clarity over criteria for completion prophylactic RHC and failure to report compliance and complications rates for patients undergoing RHC.

To determine the risk of reporting bias and the presence of small study effects, effect size estimates from the included studies were plotted against the measure of each study's size on funnel plots for each investigated parameter (Supplementary Figs. 1-14, Supplement). The visually observed asymmetry in the distribution of the funnel plots necessitated complementary tests that did not demonstrate small size effects (Supplementary Figs. 1-14, Supplement). Reasons for funnel plot asymmetry could be the fact that a small number of studies were included $(<10$ studies in several meta-analyses), heterogeneity between studies and publication bias.

\section{Pooled results for primary tumour size}

We identified seven studies reporting LN status at RHC for tumour size cutoffs of 10 and $20 \mathrm{~mm}$ [3,11-16]. The rate of LN metastases for a cutoff size $>10 \mathrm{~mm}$ was $48.6 \%$ (vs $12.1 \%$ for lesions $<10 \mathrm{~mm}$ ) among adult patients, with a random-effects OR of 4.8 (95\% CI, 1.5-15.8; heterogeneity, $P=0.061 ; I^{2}=46.3 \%$, Egger's $p$-value $=0.093$, Fig. 2a). For a cutoff size of $20 \mathrm{~mm}$, these figures were $61 \%$ (vs 


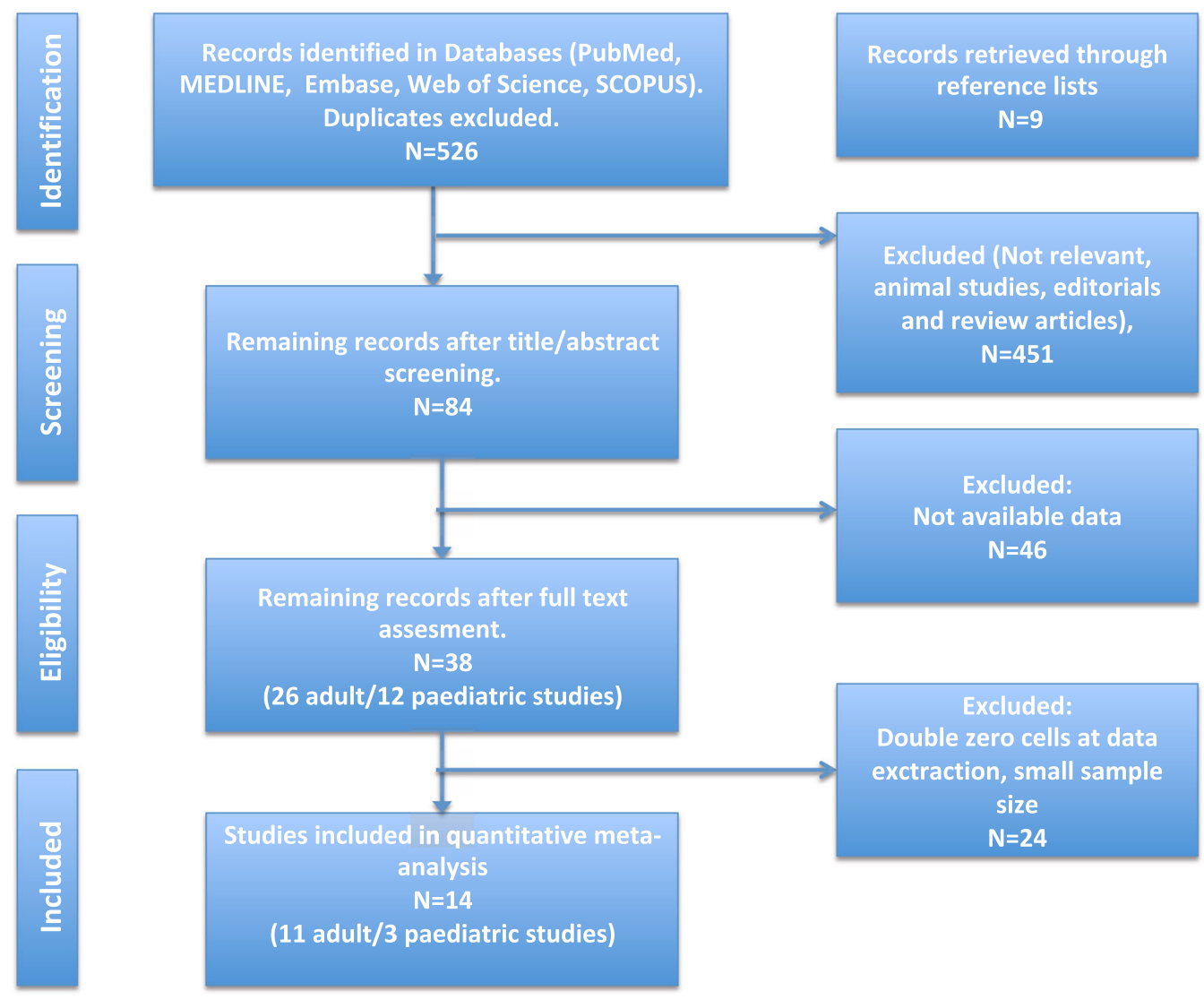

Fig. 1 PRISMA flow diagram of study selection

$28.2 \%$ for lesions $<20 \mathrm{~mm}$ ) with a random-effects OR of 3.2 (95\% CI, 1.3-7.8; heterogeneity, $P=0.020 ; I^{2}=60.1 \%$, Egger's $p$-value $=0.036$, Fig. 2b).

We also performed subgroup analysis for tumour sizes of $10-20 \mathrm{~mm}$ vs $<10 \mathrm{~mm}$ and $10-20 \mathrm{~mm}$ vs $>20 \mathrm{~mm}$ separately, to address the grey zone of $10-20 \mathrm{~mm}$ in ANEN management and avoid contamination of this subgroup by larger and smaller tumours, respectively. The rate of LN metastases for $<10 \mathrm{~mm}$ was $12.1 \%$ vs $38.5 \%$ for lesions $10-20 \mathrm{~mm}$ vs $61 \%$ for $>20 \mathrm{~mm}$ among adult patients, with a random-effects OR of 4.1 for the first comparison $(95 \% \mathrm{CI}$, 1.6-10.2; heterogeneity, $P=0.208 ; I^{2}=28.9 \%$, Egger's $p$ value $=0.120$ Fig. 3a) and of 2.2 for the second one $(95 \%$ CI, 1-4.7; heterogeneity, $P=0.120 ; I^{2}=40.6 \%$, Egger's $p$ value $=0.031$, Fig. 3b).

\section{Pooled results for tumour location}

We identified five studies reporting $\mathrm{LN}$ status at RHC in connection to ANEN location on the appendix (base vs nonbase) $[11,13,15-17]$. The rate of LN metastases in ANENs located at the base of the appendix was $25 \%$ (vs $26.7 \%$ for lesions in the body or apex of the appendix) among adult patients, with a random-effects OR of 1.4 (95\% CI, 0.4-5.2; heterogeneity, $P=0.135 ; I^{2}=43 \%$, Egger's $p$-value $=$ 0.663 Fig. 4a).

\section{Pooled results for tumour grade}

Seven studies reporting LN status at RHC relating to WD ANEN grade (Grade 1 [G1] vs Grade 2 [G1]) were included in this analysis $[3,11,13,15-18]$. The rate of $\mathrm{LN}$ metastases in G1 ANEN was $22.4 \%$ (vs $55.4 \%$ for G2 lesions) among adult patients, with a random-effects OR of 3.7 (95\% CI, 1.1-13; heterogeneity, $P=0.021 ; I^{2}=59.9 \%$; Egger's $p$-value $=0.947$; Fig. $4 \mathrm{~b}$ ).

With respect to the observed high interstudy heterogeneity in grade analysis, when considering potential effect modifiers in univariate metagression analysis, there were significant effects of size and other morphological parameters (tumour size 10-20 mm $P>|z|$ : 0.012; location, base $P>|z|$ : 0.026; meso-appendiceal invasion $P>|z|$ : 0.044; lymph vessel invasion $P>|z|$ : 0.039 ; perineural invasion $P$ $>|z|$ : 0.049), suggesting that more than one parameters may impact the risk for $\mathrm{LN}$ positivity in Grade 2 patients, e.g. in the grey zone of $10-20 \mathrm{~mm}$ tumour size. 


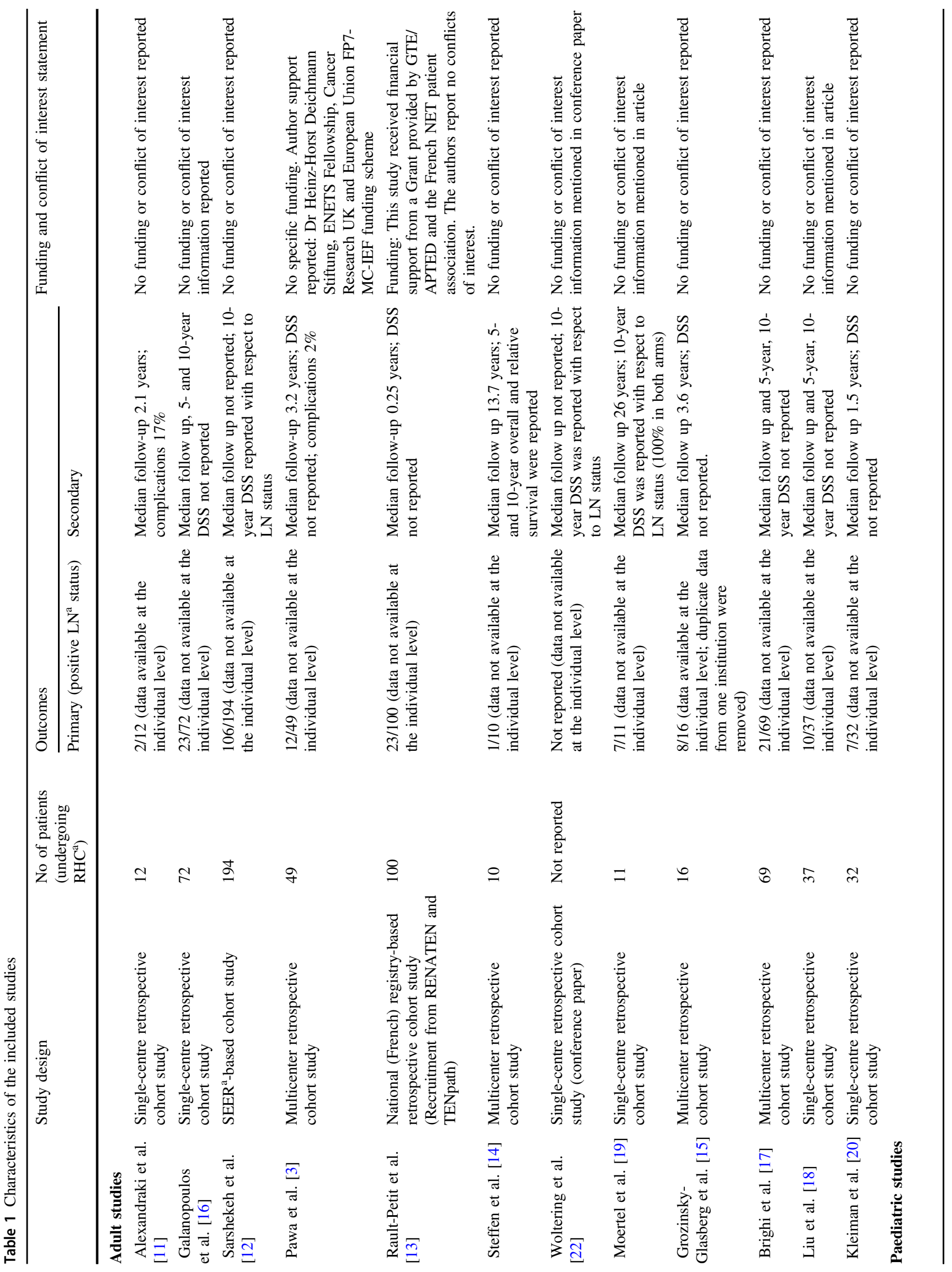




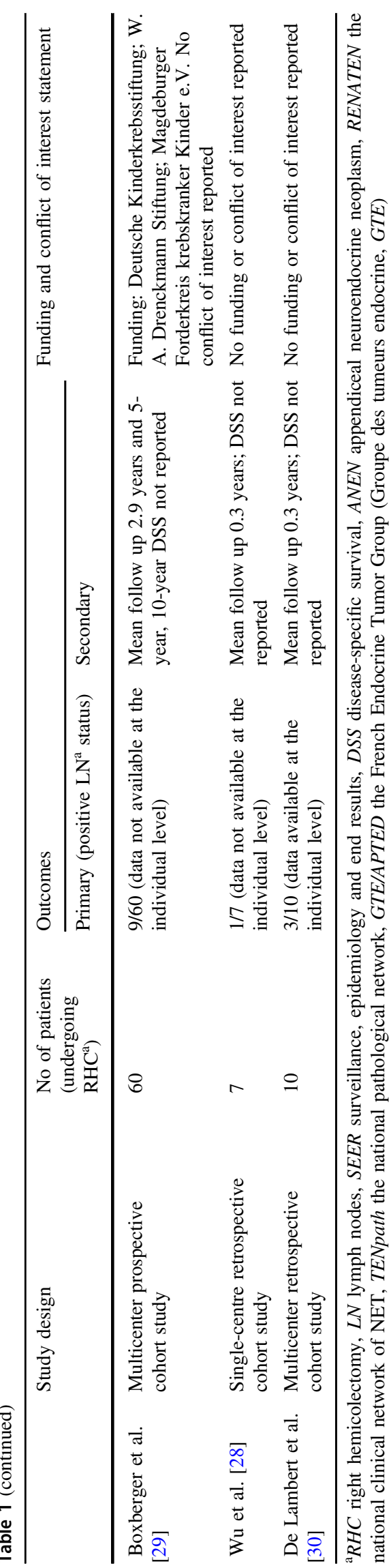

Table 2 Newcastle-Ottawa scale (NOS) cohort star template

Selection Comparability Exposure

Adult studies

Alexandraki et al. [11]

Galanopoulos et al. [16]

Sarshekeh et al. [22]

Pawa et al. [3]

Rault-Petit et al. [13]

Steffen et al. [14]

Woltering et al. [22]

Moertel et al. [19]

Grozinsky-Glasberg et al. [15]

Brighi et al. [17]

Liu et al. [18]

Kleiman et al. [20]

Paediatric studies

Boxberger et al. [29]

Wu et al. [28]

De Lambert et al. [30]

Pooled results for other morphological parameters (meso-appendix, vascular, lymph vessel and perineural invasion)

Seven studies reporting LN status at RHC in connection to meso-appendix invasion were included $[11,13,14,16,17$, 19, 20]. The rate of LN metastases in adult patients demonstrating meso-appendix invasion was $30.3 \%$ (vs $26.2 \%$ in adult patients without meso-appendix invasion), with a random-effects OR of 1.4 (95\% CI, 0.8-2.4; heterogeneity, $P=0.43 ; I^{2}=0 \%$, Egger's $p$-value $=0.607$, Fig. 5 a).

In addition, we identified four adult studies reporting $\mathrm{LN}$ status at RHC in connection to vascular invasion $[15,16,19,20]$. The rate of $\mathrm{LN}$ metastases in these patients was $60 \%$ (vs $26.7 \%$ in patients without vascular invasion), with a random-effects OR of 4.3 (95\% CI, 1.7-11; heterogeneity, $P=0.974 ; I^{2}=0 \%$, Egger's $p$-value $=0.095$, Fig. $5 \mathrm{~b})$. For other morphological parameters, such as lymph vessel and perineural invasion, we identified three $[13,17,21]$ and four eligible adult studies $[11,13,15,16]$, respectively. The rate of $\mathrm{LN}$ metastases in patients demonstrating lymph vessel invasion was $45.6 \%$ (vs $21.6 \%$ in patients without lymph vessel invasion), with a randomeffects OR of 3.4 (95\% CI, 1.7-6.8; heterogeneity, $P=$ $0.954 ; I^{2}=0 \%$, Egger's $p$-value $=0.690$, Fig. 6a). These figures for ANEN with perineural invasion were: LN metastases rate $56.8 \%$ (vs $19 \%$ in patients without perineural invasion) with a random-effects OR of 5.8 (95\% CI, 1.8-18.2; heterogeneity, $P=0.255 ; I^{2}=26.2 \%$, Egger's $p$ value $=0.065$, Fig. 6b) . 
Study

b

Study

ID
Events, Size Events,

$>20 \mathrm{~mm} \& \quad$ Size $\%$

$10-20 \mathrm{~mm} \quad<10 \mathrm{~mm} \quad$ Weight

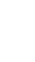

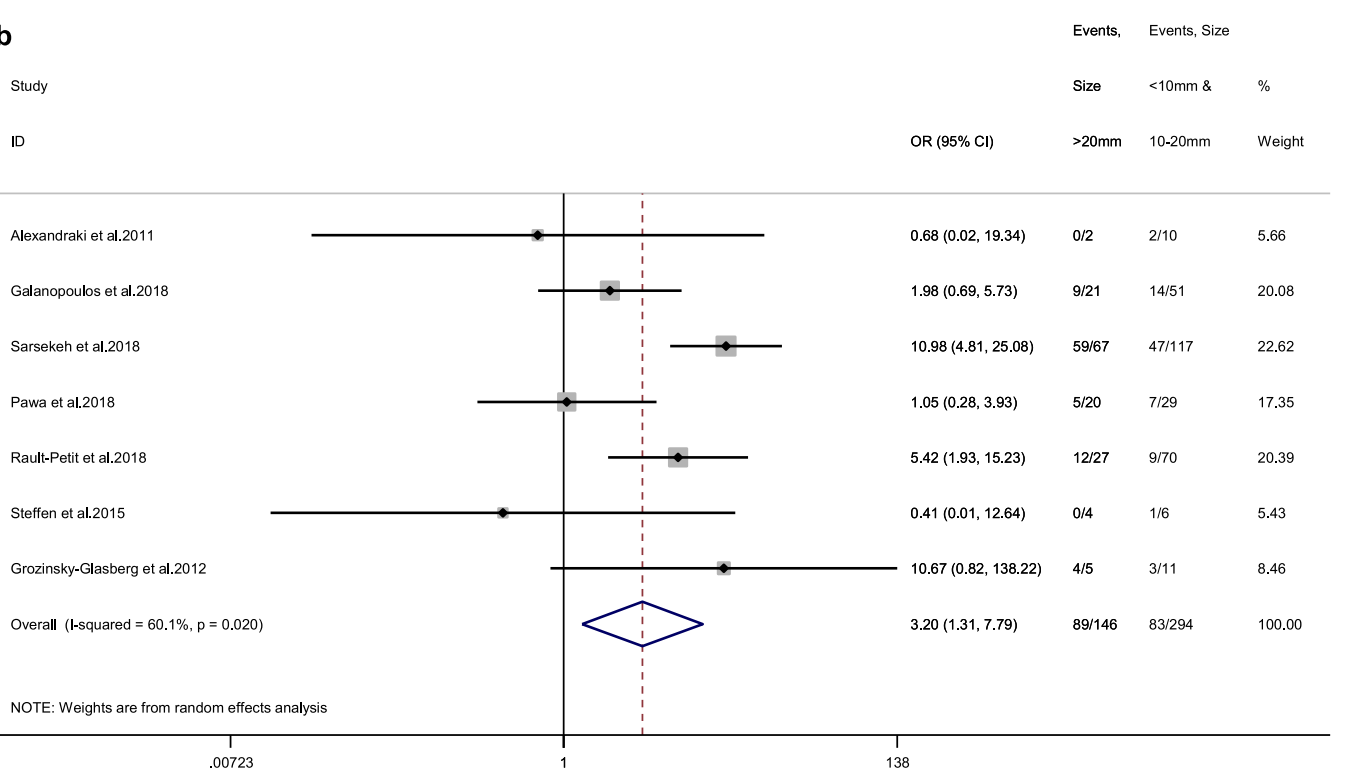

Fig. 2 a Forest plot comparing the rate of $\mathrm{LN}$ metastases at RHC and at a $10 \mathrm{~mm}$ size cutoff, i.e. in tumour size $>10 \mathrm{~mm}$ vs tumour size $<10 \mathrm{~mm}$. b Forest plot comparing the rate of LN metastases at RHC at

\section{Pooled results for disease-specific survival (DSS) and complications}

Only two studies reported 10-year DSS stratified by LN status at RHC and these two were meta-analysed [12, 22]. The 10-year DSS rate for adult patients without LN metastases was $99.2 \%$ compared with $95.6 \%$ for patients with $\mathrm{LN}$ undergoing RHC (random-effects OR of 0.2 , 95\% CI, 0.02-2.4; heterogeneity, $P=0.144 ; I^{2}=53.1 \%$, Fig. 7). In seven studies reporting 5-year DSS rates following RHC versus appendectomy alone, $100 \%$ DSS was demonstrated in both arms (RHC vs appendectomy alone) [14, 19, 23-26].

The rate of complications that was encountered in patients undergoing prophylactic RHC was reported in four a $20 \mathrm{~mm}$ size cutoff, i.e. in tumour size $>20 \mathrm{~mm}$ vs tumour size $<20 \mathrm{~mm}$. Meta-analysis of all studies carried out using a randomeffects model; Odds ratios are shown with $95 \%$ confidence intervals

studies $[3,11,25,27]$ and was as high as $11.4 \%$. However, a lower complication rate was demonstrated over time when comparing the studies by the year of publication (chisquare, $P<0.0001)$. The complications' severity ranged significantly, although it's range was not appropriately classified.

\section{Pooled results for morphological parameters in paediatric patients}

The rate of $\mathrm{LN}$ metastases for a cutoff size $>10 \mathrm{~mm}$ was $17.9 \%$ (vs $15 \%$ for lesions $<10 \mathrm{~mm}$ ) among paediatric patients, with a random-effects OR of 1.3 (95\% CI, 0.3-5.9; heterogeneity, $P=0.645 ; I^{2}=0 \%$, Begg's $p$-value $=0.317$, 


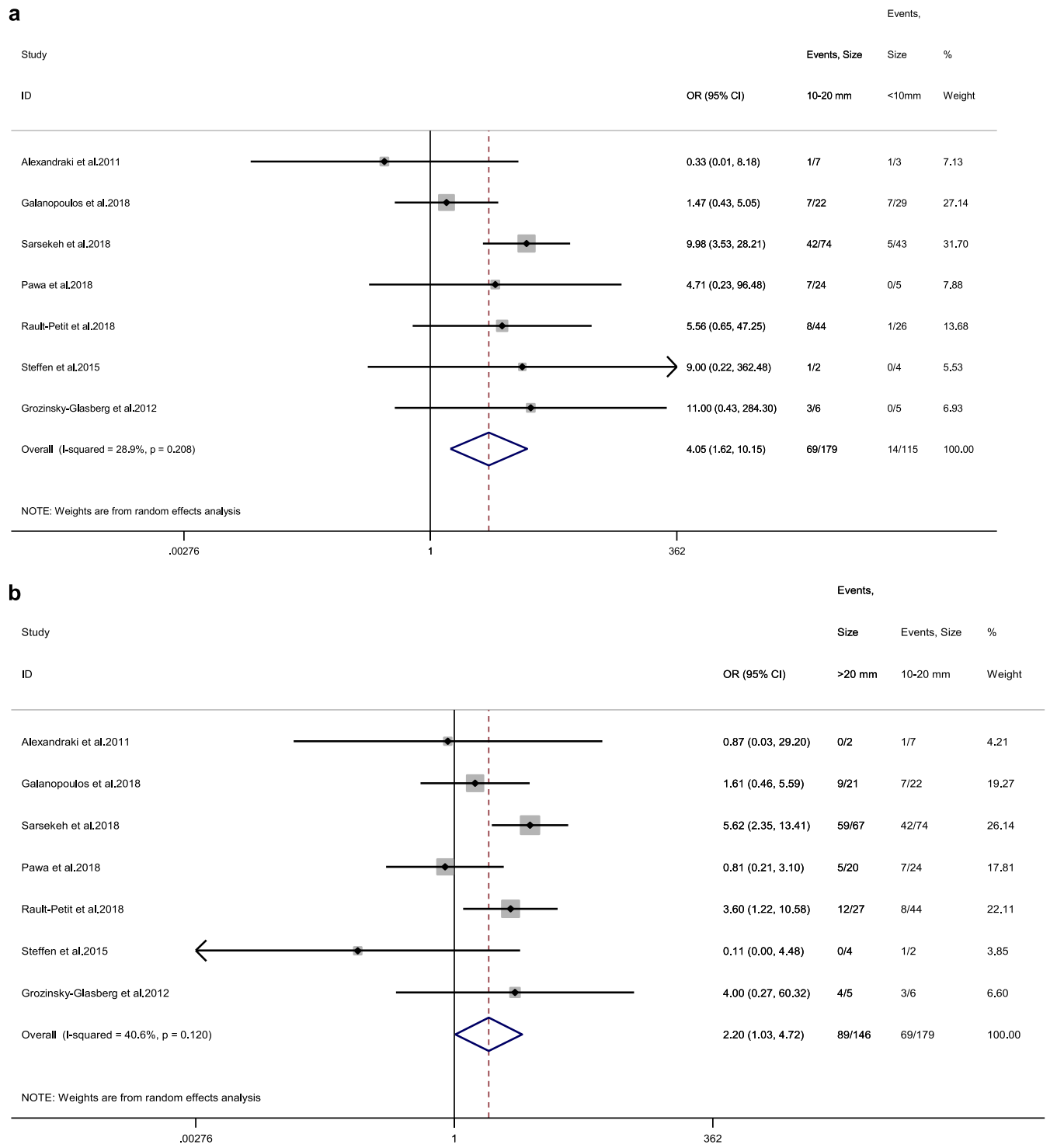

Fig. 3 a Forest plot comparing the rate of LN metastases at RHC in tumours $<10 \mathrm{~mm}$ vs tumours between $10-20 \mathrm{~mm}$. b Forest plot comtumours between 10-20 mm. Meta-analysis of all studies carried out using a random-effects model; Odds ratios are shown with $95 \%$ confidence interval

Supplementary Fig. 8A) [28, 29]. For meso-appendix invasion, the rate of $\mathrm{LN}$ metastases was $16.7 \%$ in patients with this invasion (vs $22.2 \%$ in patients without), with a random-effects OR of 0.8 (95\% CI, 0.1-5; heterogeneity, $P$ $=0.234 ; I^{2}=31.1 \% ;$ Egger's $p$-value $=0.628 ;$ Begg's $p$ value $=0.602$; Fig. 8 b) $[28-30]$. Finally, the rate of LN metastases was $28.6 \%$ in paediatric patients with lymph vessel invasion (vs $13.2 \%$ in patients without), with a random-effects OR of 2.8 (95\% CI, 0.5-14.9; heterogeneity, $P=0.625 ; \quad I^{2}=0 \% ;$ Begg's $p$-value $=0.317 ;$ Fig. 8 c) $[28,29]$. The trim and fill methods were not applied due to lack of heterogeneity in the included paediatric studies. In three studies reporting 10-year DSS rates following RHC vs appendectomy alone, 100\% DSS was demonstrated in both arms (RHC vs appendectomy alone) [31-33]. In addition, three more studies were identified reporting 5-year DSS of $100 \%$ irrespective of the extent of the surgical procedure undertaken [34-36].

Generally, in paediatric studies, available data were limited and not always appropriately reported. Meta-analysis was not feasible for tumour size cutoff $>20 \mathrm{~mm}$ as only one study was eligible $(\mathrm{OR}=6.080,95 \%$ CI: $1.213-30.473)$ [29]. One study only addressed grade (OR: $1.889,95 \% \mathrm{CI}$ : 0.050-72.022) and vascular invasion (OR: $0.37595 \% \mathrm{CI}$ : 0.022-6.348) in paediatric patients [30]. Data on complications secondary to prophylactic RHC were not available. 
Study

ID

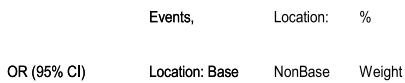

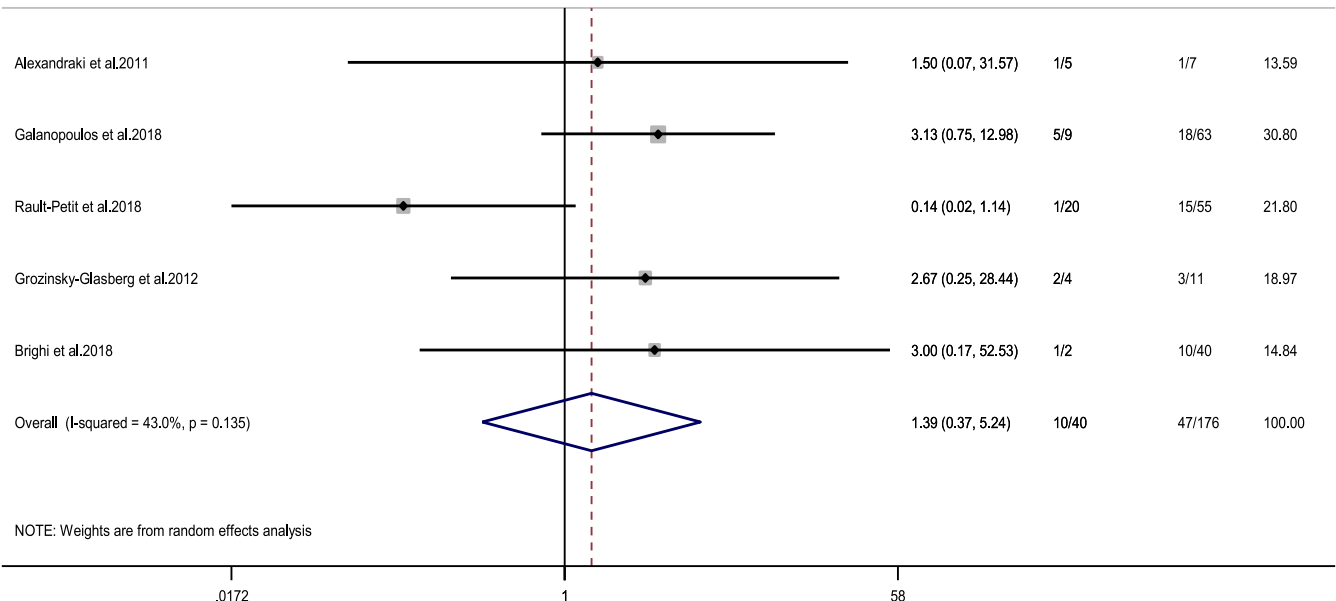

b

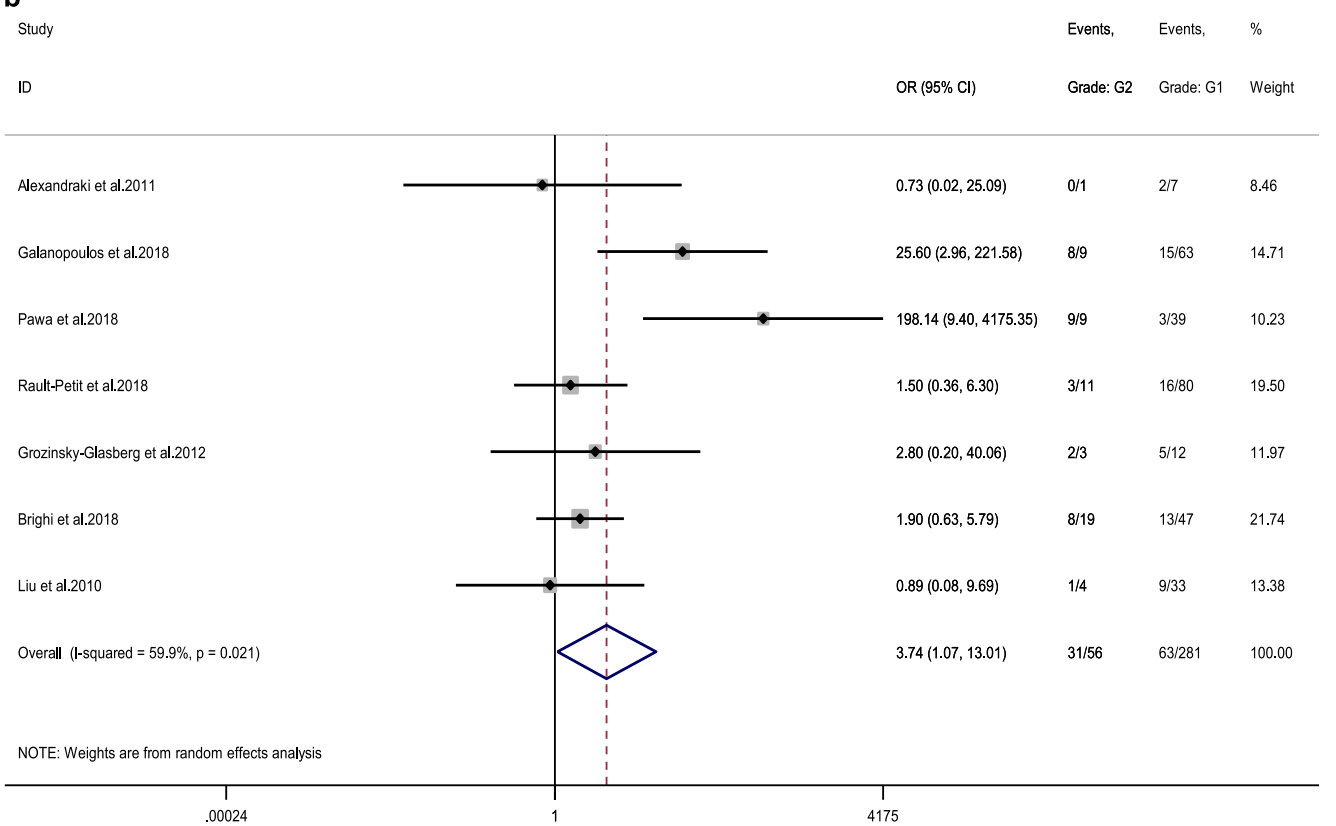

Fig. 4 a Forest plot comparing the rate of LN metastases at RHC in tumours located in the appendix base vs tumours located in the body or tail of the appendix. b Forest plot comparing the rate of LN metastases

at RHC in Grade 1 vs Grade 2 ANEN. Meta-analysis of all studies carried out using a random-effects model; Odds ratios are shown with $95 \%$ confidence intervals

\section{Discussion}

The present meta-analysis confirms that tumour size, vascular invasion, lymph vessel invasion and perineural invasion are strong predictors for LN metastases in adult patients with ANEN. On the contrary, tumour location on the appendix as well as meso-appendix invasion were not unambiguously confirmed as affecting the risk for LN metastases. Regarding different tumour size cutoffs in adult patients, the rate of $\mathrm{LN}$ metastases at RHC for lesions $<10 \mathrm{~mm}$ was $12.1 \%$ vs $38.5 \%$ for lesions
$10-20 \mathrm{~mm}$ vs $61 \%$ for tumours $>20 \mathrm{~mm}$. A random-effects OR of 4.8 (95\% CI, 1.5-15.8) was estimated for a $10 \mathrm{~mm}$ size cutoff, whereas for a $20 \mathrm{~mm}$ cutoff, random-effects OR was 3.2 (95\% CI, 1.3-7.8). Thus, primary tumour size retains its value as a predictive marker of metastatic propensity to locoregional $\mathrm{LN}$.

Disease-specific mortality rates at 10 years of follow-up reported in two studies of adults with ANEN were as low as $0.8 \%$ and $4.4 \%$ for patients with and without $\mathrm{LN}$ metastases undergoing RHC, respectively. Notably, the presence of LN metastases does not seem to clearly affect survival in 


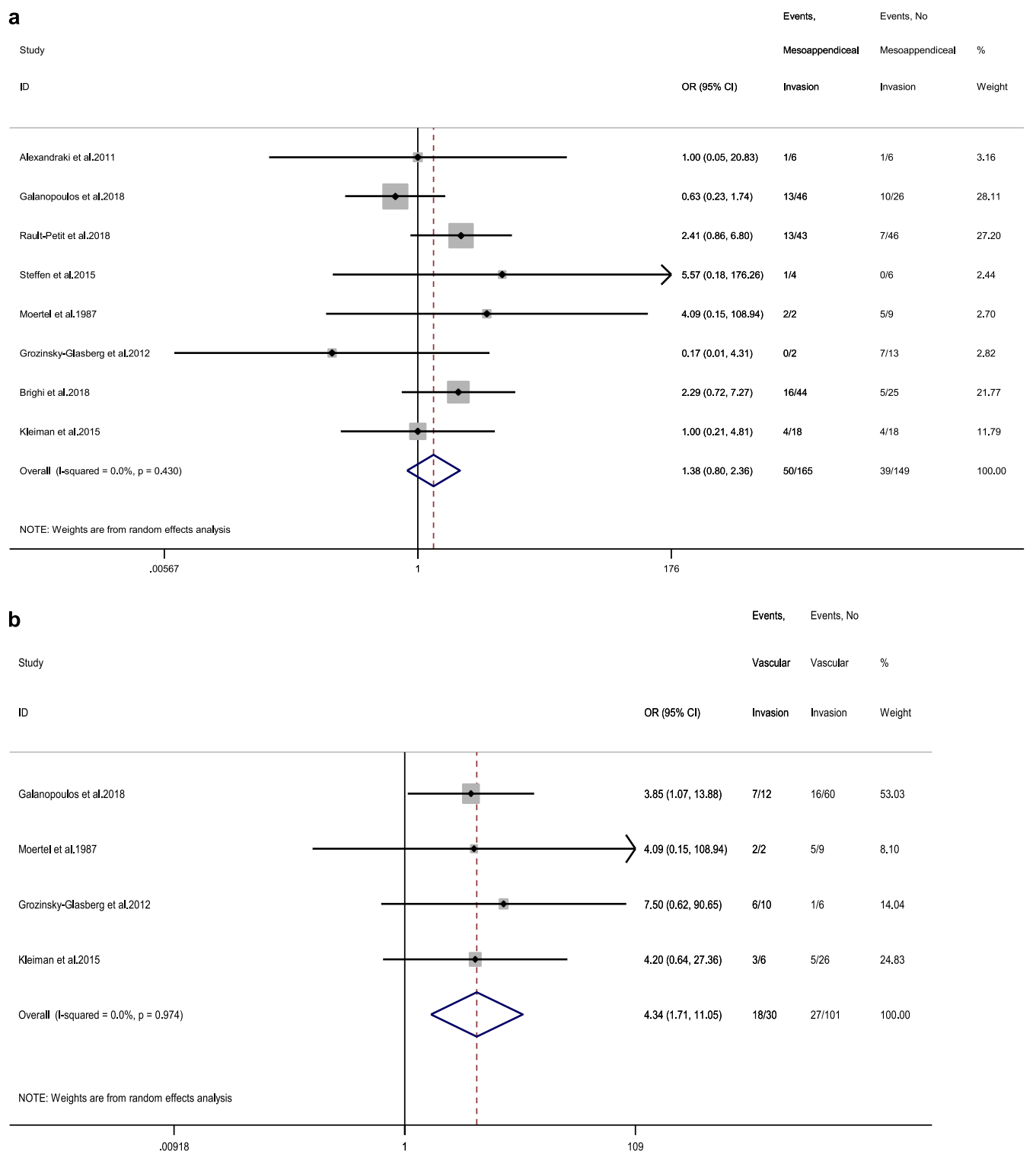

Fig. 5 a Forest plot comparing the rate of $\mathrm{LN}$ metastases at RHC in tumours with meso-appendiceal invasion vs tumours without invasion of the meso-appendix. b Forest plot comparing the rate of LN metastases at RHC in tumours with vascular invasion vs tumours

without vascular invasion. Meta-analysis of all studies carried out using a random-effects model; Odds ratios are shown with $95 \%$ confidence interval

patients who have undergone curative resection, i.e. either RHC or appendectomy alone. Thus, we were unable to provide evidence that the presence of $\mathrm{LN}$ metastases affects OS or that RHC exerts a prophylactic effect. The complication rate of prophylactic RHC in this meta-analysis was $11.4 \%$, with lower complication rates demonstrated over time, implying a generally safer procedure nowadays. In paediatric patients, available data were limited and no clear relationships between the parameters we investigated and LN metastases were evident. However, regardless of the extent of surgery in children, favourable long-term survival rates were reported, implying an excellent prognosis for paediatric ANEN cases subjected to curative resection.
In the efforts to adopt a precision medicine surgical and post-treatment surveillance approach in the management of ANEN, one of the main challenges is related to the paucity of validated biomarkers to guide the extent of locoregional resective surgery, i.e. to select the best candidates for prophylactic RHC and monitor patient outcomes. Overall, the rate of LN metastases in our study prior to different strata division and analysis, in both adult and pediatric patients, was $34.6 \%$, as compared with the corresponding figure of 24.5\% in a recent meta-analysis in ANENs [37]. The reasons for this discrepancy may be multifaceted and could potentially imply that the implementation of not validated risk factors, such as meso-appendiceal invasion in the grey 


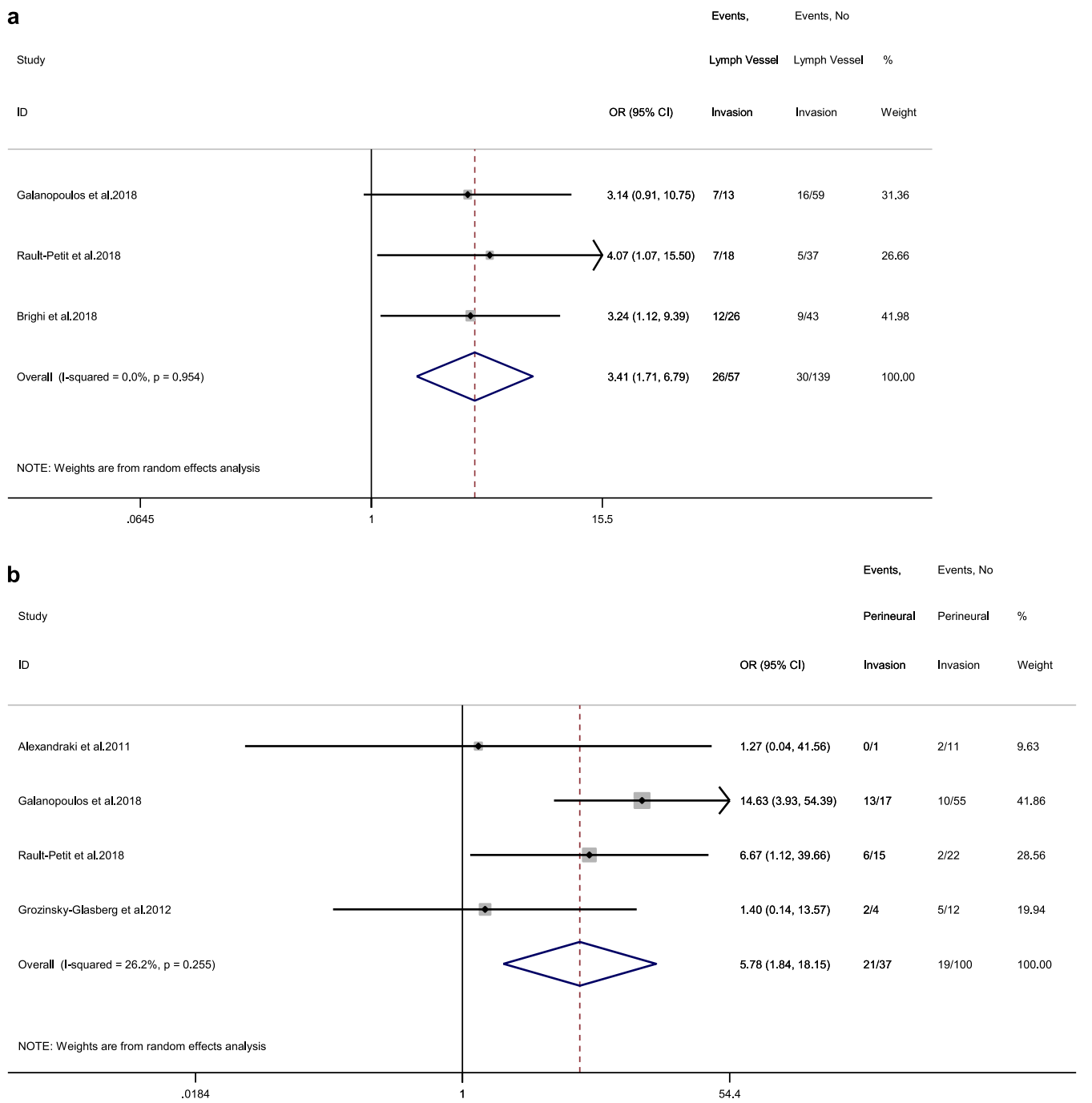

Fig. 6 a Forest plot comparing the rate of $\mathrm{LN}$ metastases at RHC in tumours with lymph vessel invasion vs tumours without lymph vessel invasion. b Forest plot comparing the rate of $\mathrm{LN}$ metastases at RHC in tumours with perineural invasion vs tumours without perineural invasion. Meta-analysis of all studies carried out using a randomeffects model; Odds ratios are shown with $95 \%$ confidence interval zone of $10-20 \mathrm{~mm}$ tumour size to guide the extent of surgery may have led to unnecessary completion RHCs.

We conducted an NOS quality assessment on all the included studies (Table 2). All cohort studies scored at least five points, achieving moderate to high quality. Heterogeneity between studies was observed in the assessment of certain parameters, i.e. lesion size cutoff of $20 \mathrm{~mm}$, grade and mortality rate. As statistically significant "positive" results are more likely to be published rapidly in English language and high impact journals, leading to reporting biases, we also assessed non-English studies, as well as unpublished data from conference papers [21, 22]. Complementary testing did not reveal small study effects in the present meta-analysis. Further factors, such as confounding and the precision of measurements, e.g. ICD-coded data in registry-based studies, might have introduced bias [38]. Importantly, although registry-based studies might differ regarding the strength of their evidence, they still constitute a valuable information source for evidence-based medicine [9].

With regard to the tumour size cutoffs investigated in this meta-analysis, we should clarify that traditionally a tumour size $>20 \mathrm{~mm}$ constitutes a general indication for more extensive surgery, commonly completion RHC, based on the seminal paper in the New England Journal of Medicine published by pioneers in the field, namely Moertel et al. in 1987 [19]. To date, for ANENs between 10 and $20 \mathrm{~mm}$, various morphological parameters at histopathology have been applied as per ENETS guidelines to identify patients who would potentially benefit from completion prophylactic RHC. Therefore, for lesions $<20 \mathrm{~mm}$ subjected to RHC, one or more morphological parameter was presumably present at histopathology in order to qualify for completion surgery. However, few studies reported patient data at the 
a

Study

ID
Events,

Mesoappendiceal Mesoappendiceal \%

R $(95 \% \mathrm{Cl})$

Invasion

Invasion Weight

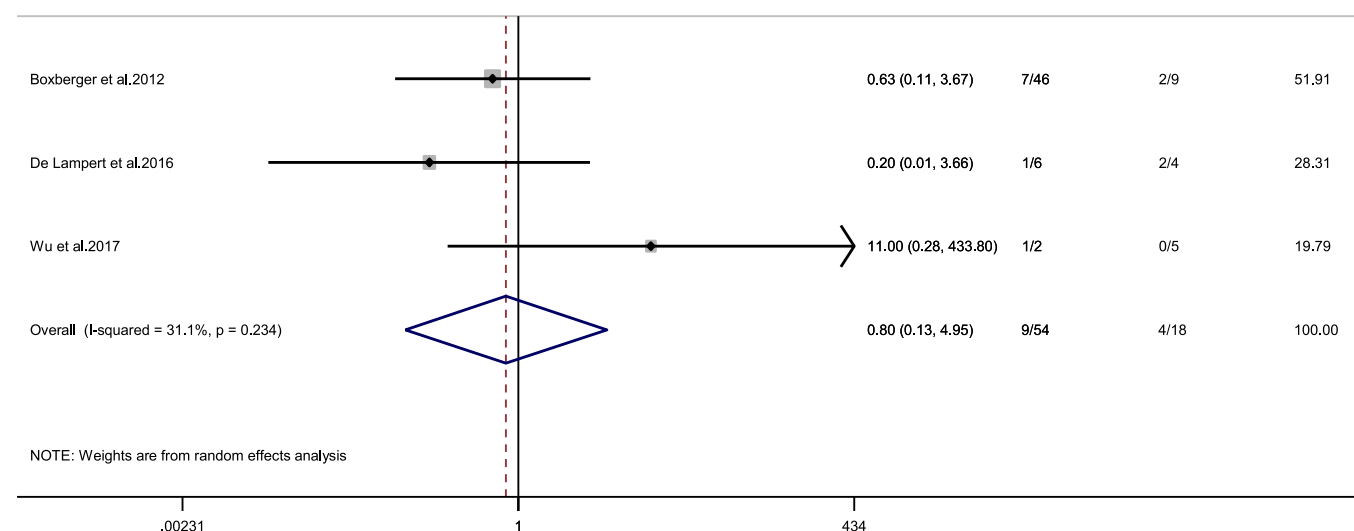

b

Study

ID

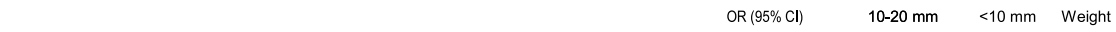

NOTE: Weights are from random effects analysis

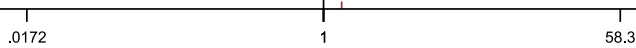

8.3

c

Study

ID

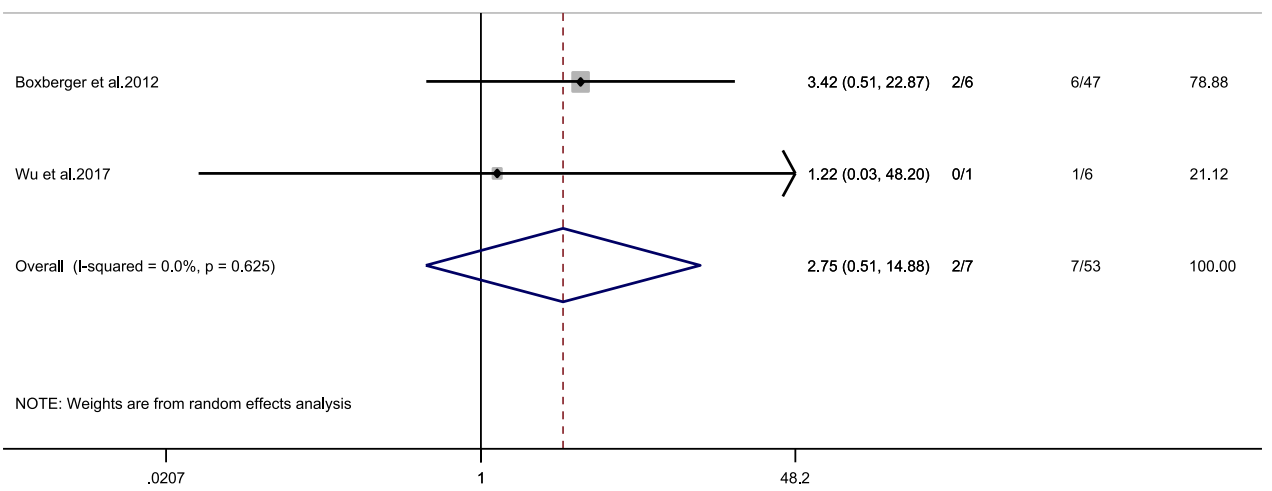

Fig. 8 a Forest plot comparing the rate of LN metastases at RHC and at a $10 \mathrm{~mm}$ size cutoff, i.e. in tumour size $>10 \mathrm{~mm}$ vs tumour size $<10 \mathrm{~mm}$ for paediatric patients. b Forest plot comparing the rate of LN metastases at RHC in tumours with meso-appendiceal invasion vs tumours without invasion of the meso-appendix in paediatric patients. c Forest plot comparing the rate of LN metastases at RHC in tumours with lymph vessel invasion vs tumours without lymph vessel invasion in paediatric patients. Meta-analysis of paediatric studies carried out using a random-effects model; Odds ratios are shown with $95 \%$ confidence interval 


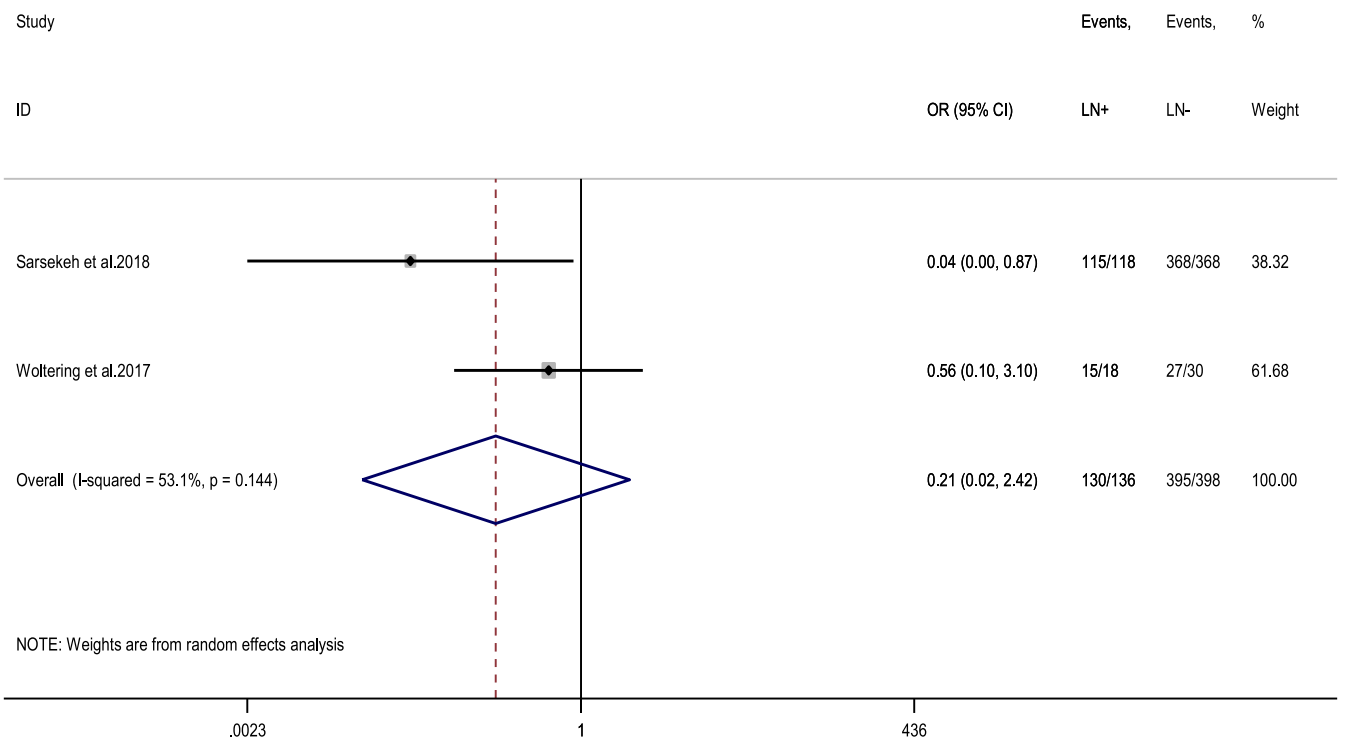

Fig. 7 Forest plot comparing 10-year disease-specific survival in ANEN patients with LN metastases vs ANEN patients without LN metastases. Meta-analysis of all studies carried out using a random-effects model; Odds ratios are shown with 95\% confidence intervals

individual level and in some there was ambiguity over criteria for completion prophylactic RHC. Thus, the rates and OR of LN metastases in ANEN $<20 \mathrm{~mm}$ must be interpreted in the light of this knowledge.

The prognostic significance of meso-appendix invasion was not confirmed in our study. In the current ENETS guidelines, a meso-appendix invasion depth of $>3 \mathrm{~mm}$ is arbitrarily used to distinguish $\mathrm{T} 2$ from $\mathrm{T} 3$ tumours in the TNM classification. However, there is insufficient information in the literature to substantiate whether mesoappendix invasion and the $3 \mathrm{~mm}$ cutoff are validated prognostic factors to guide the extent of surgery. In addition, the location of the primary tumour in the appendix has been implied as a risk factor for non-radical resection at appendectomy, thus metastatic propensity necessitating completion RHC. However, this was not confirmed in our study, as no clear association with the presence of LN metastases was evident. Finally, Grade 2 was potentially linked to higher risk for LN metastases; however, due to high interstudy heterogeneity and potential effect modifiers evident in metagression analysis, no safe conclusions could be derived. On the contrary, vascular, lymph vessel and perineural invasion were substantiated as strong predictors for LN metastases in adult patients with ANEN.

ANEN's clinical course in children may be completely benign, as paediatric studies report 5- and 10-year DSS of $100 \%$ in all patients who had undergone curative resection, i.e. appendectomy alone or completion RHC. However, due to insufficient data having been reported, our meta-analysis could not confirm predictive factors for LM metastases to guide the extent of surgery as in adult patients. For tumour size cutoff $>20 \mathrm{~mm}$, a single study was eligible and reported an OR as high as 6.1, (95\% CI: 1.2-30.4) for LN metastases. However the clinical significance of this remains unclear and should be validated in more studies [29].

Our study has some limitations. Importantly, it constitutes an unplanned subset analysis of multiple observational studies on the rare entity of ANEN. The eligible institutional studies were underpowered or not even designed to assess differences in the outcomes of our interest. Further limitations include a lack of centralized pathology review that not only concerns interstudy concordance for tumour size and other histopathological parameters, but also changes within the NEN grading system over time. Moreover, regarding the SEER data quality, it should be noted that complete pathological data were not routinely included in the SEER database prior to 1988. However, in the SEER report by Sarshekeh included in this meta-analysis study, participants were only eligible for inclusion after 1988. All these limitations concern the general applicability of older studies nowadays, yet robust effects (e.g. for vascular invasion and the $20 \mathrm{~mm}$ size cutoff) had already been observed in the seminal study by Moertel et al. 32 years ago, as well as in very recent reports. Another important limitation was the high interstudy heterogeneity encountered when assessing certain morphological parameters, i.e. grade. However, in the absence of prospective studies, we used the best available evidence and applied a comprehensive search strategy and advanced statistical methods, including meta-regression, demonstrating the risk of LN metastases in relation to histopathology and their prognostic significance in adult and paediatric patients with WD ANEN.

In the random-effects model we adopted for 10-year DSS, $I^{2}$ was $53 \%$, suggesting that substantial variability in 
effect estimates on LN status is due to real study differences (heterogeneity). This is also evident from the wide scatter of effect estimates with broad CI that depict the uncertainty around this pooled estimate. Therefore, there is no strong evidence to date that positive locoregional LN metastases are associated with higher mortality. Hence, the true impact of LN status in ANEN, and whether a prophylactic surgical approach is beneficial, both need to be elucidated in further studies with long-term (>10 years) follow-up.

\section{Conclusions}

Our study provides a systematic review and quantitative meta-analysis of ANEN summarizing all available evidence and confirming previously established prognostic factors. It also highlights areas of future research needed in the field. In addition, we confirmed that tumour size $>20 \mathrm{~mm}$, as well as $>10 \mathrm{~mm}$ in the presence of vascular, lymph vessel or perineural invasion, are associated with an increased risk for LN metastases in adult patients with ANEN. In paediatric studies, available information is limited and no strong morphological predictors for LN metastases could be confirmed. ANENs have a generally favourable prognosis with rather low disease-specific mortality rates, as demonstrated in the adult studies included in the meta-analysis. Survival does not seem to be significantly depreciated after curative ANEN resection with either RHC or appendectomy alone. In addition, paediatric ANEN prognosis is excellent when subjected to curative resection. Longitudinal studies with $>10$ years follow-up are warranted to determine the true impact of LN status in patient survival from real-life data and whether a prophylactic surgical approach with completion RHC based on the findings of the present metaanalysis would be beneficial.

Funding Open access funding provided by Uppsala University. K.D. was supported by the Swedish Society of Medicine Post Doctoral Scholarship and the Lennanders Fund. The other authors of the study have not received any funding.

\section{Compliance with ethical standards}

Conflict of interest The authors declare that they have no conflict of interest.

Publisher's note Springer Nature remains neutral with regard to jurisdictional claims in published maps and institutional affiliations.

Open Access This article is distributed under the terms of the Creative Commons Attribution 4.0 International License (http://crea tivecommons.org/licenses/by/4.0/), which permits unrestricted use, distribution, and reproduction in any medium, provided you give appropriate credit to the original author(s) and the source, provide a link to the Creative Commons license, and indicate if changes were made.

\section{References}

1. M. Lundqvist, E. Wilander, Subepithelial neuroendocrine cells and carcinoid tumours of the human small intestine and appendix. A comparative immunohistochemical study with regard to serotonin, neuron-specific enolase and S-100 protein reactivity. J. Pathol. 148(2), 141-147 (1986). https://doi.org/10.1002/path. 1711480204

2. C.G. Moertel, M.B. Dockerty, E.S. Judd, Carcinoid tumors of the vermiform appendix. Cancer 21(2), 270-278 (1968)

3. N. Pawa, A.K. Clift, H. Osmani, P. Drymousis, A. Cichocki, R. Flora, R. Goldin, D. Patsouras, A. Baird, A. Malczewska, J. Kinross, O. Faiz, A. Antoniou, H. Wasan, G.A. Kaltsas, A. Darzi, J.B. Cwikla, A. Frilling, Surgical management of patients with neuroendocrine neoplasms of the appendix: appendectomy or more. Neuroendocrinology 106(3), 242-251 (2018). https://doi. org/10.1159/000478742

4. S.S. Groth, B.A. Virnig, W.B. Al-Refaie, S.L. Jarosek, E.H. Jensen, T.M. Tuttle, Appendiceal carcinoid tumors: predictors of lymph node metastasis and the impact of right hemicolectomy on survival. J. Surg. Oncol. 103(1), 39-45 (2011). https://doi.org/10. $1002 /$ jso. 21764

5. M.L. McGory, M.A. Maggard, H. Kang, J.B. O’Connell, C.Y. Ko, Malignancies of the appendix: beyond case series reports. Dis. Colon Rectum 48(12), 2264-2271 (2005). https://doi.org/10.1007/ s10350-005-0196-4

6. U.F. Pape, B. Niederle, F. Costa, D. Gross, F. Kelestimur, R. Kianmanesh, U. Knigge, K. Oberg, M. Pavel, A. Perren, C. Toumpanakis, J. O'Connor, E. Krenning, N. Reed, D. O'Toole; Vienna consensus conference participants, ENETS consensus guidelines for neuroendocrine neoplasms of the appendix (excluding goblet cell carcinomas). Neuroendocrinology 103(2), 144-152 (2016). https://doi.org/10.1159/000443165

7. C. Toumpanakis, N. Fazio, E. Tiensuu Janson, D. Horsch, A. Pascher, N.,D.,O.A.T. Reed, E. Nieveen van Dijkum, S. Partelli, A. Rinke, B. Kos-Kudla, F. Costa, U.F. Pape, S. GrozinskyGlasberg, J.Y. Scoazec; E.M.A.B. participants, Unmet needs in appendiceal neuroendocrine neoplasms. Neuroendocrinology 108 (1), 37-44 (2019). https://doi.org/10.1159/000493894

8. D. Moher, A. Liberati, J. Tetzlaff, D.G. Altman, P. Group, Preferred reporting items for systematic reviews and meta-analyses: the PRISMA statement. Ann. Intern. Med. 151(4), 264-269, W264 (2009)

9. T. Mathes, D. Pieper, Study design classification of registry-based studies in systematic reviews. J. Clin. Epidemiol. 93, 84-87 (2018). https://doi.org/10.1016/j.jclinepi.2017.09.016

10. A. Stang, Critical evaluation of the Newcastle-Ottawa scale for the assessment of the quality of nonrandomized studies in metaanalyses. Eur. J. Epidemiol. 25(9), 603-605 (2010). https://doi. org/10.1007/s10654-010-9491-Z

11. K.I. Alexandraki, J. Griniatsos, K.I. Bramis, N. Ballian, N. Dimitriou, T. Giannakakis, C. Tsigris, E. Felekouras, G.A. Kaltsas, Clinical value of right hemicolectomy for appendiceal carcinoids using pathologic criteria. J. Endocrinol. Investig. 34(4), 255-259 (2011). https://doi.org/10.1007/bf03347081

12. A.M. Sarshekeh, S. Advani, D.M. Halperin, C. Conrad, C. Shen, J.C. Yao, A. Dasari, Regional lymph node involvement and outcomes in appendiceal neuroendocrine tumors: a SEER database analysis. Oncotarget 8(59), 99541-99551 (2017). https://doi.org/ 10.18632/oncotarget.20362

13. B. Rault-Petit, C. Do Cao, S. Guyetant, R. Guimbaud, V. Rohmer, C. Julie, E. Baudin, B. Goichot, R. Coriat, A. Tabarin, J. Ramos, P. Goudet, V. Hervieu, J.Y. Scoazec, T. Walter, Current management and predictive factors of lymph node metastasis of appendix neuroendocrine tumors: a national study from the french 
group of endocrine tumors (GTE). Ann. Surg., (2018). https://doi. org/10.1097/sla.0000000000002736

14. T. Steffen, S.M. Ebinger, R. Warschkow, C. Luthi, B.M. Schmied, T. Clerici, Long-term survival is not impaired after the complete resection of neuroendocrine tumors of the appendix. World J. Surg. 39(11), 2670-2676 (2015). https://doi.org/10.1007/s00268015-3164-8

15. S. Grozinsky-Glasberg, K.I. Alexandraki, D. Barak, V. Doviner, P. Reissman, G.A. Kaltsas, D.J. Gross, Current size criteria for the management of neuroendocrine tumors of the appendix: are they valid? Clinical experience and review of the literature. Neuroendocrinology 98(1), 31-37 (2013). https://doi.org/10.1159/ 000343801

16. M. Galanopoulos, R. McFadyen, I. Drami, R. Naik, N. Evans, T. V. Luong, J. Watkins, M. Caplin, C. Toumpanakis, Challenging the current risk factors of appendiceal neuroendocrine neoplasms: can they accurately predict local lymph nodal invasion? Results from a large case series. Neuroendocrinology, 1-8 (2019). https:// doi.org/10.1159/000499381

17. N. Brighi, S. La Rosa, G. Rossi, F. Grillo, S. Pusceddu, M. Rinzivillo, F. Spada, S. Tafuto, S. Massironi, A. Faggiano, L. Antonuzzo, D. Santini, F. Sessa, R. Maragliano, F. Gelsomino, M. Albertelli, C. Vernieri, F. Panzuto, N. Fazio, C. De Divitiis, G. Lamberti, A. Colao, G.D. Fave, D. Campana, Morphological factors related to nodal metastases in neuroendocrine tumors of the appendix: a multicentric retrospective study. Ann. Sur., (2018). https://doi.org/10.1097/sla.0000000000002939

18. E. Liu, D.A. Telem, J. Hwang, R.R. Warner, A. Dikman, C.M. Divino, The clinical utility of Ki-67 in assessing tumor biology and aggressiveness in patients with appendiceal carcinoids. J. Surg. Oncol. 102(4), 338-341 (2010). https://doi.org/10.1002/jso. 21634

19. C.G. Moertel, L.H. Weiland, D.M. Nagorney, M.B. Dockerty, Carcinoid tumor of the appendix: treatment and prognosis. N. Engl. J. Med. 317(27), 1699-1701 (1987). https://doi.org/10. 1056/nejm198712313172704

20. D.A. Kleiman, B. Finnerty, T. Beninato, R. Zarnegar, G. Nandakumar, T.J. Fahey 3rd, S.W. Lee, Features associated with metastases among well-differentiated neuroendocrine (carcinoid) tumors of the appendix: the significance of small vessel invasion in addition to size. Dis. Colon Rectum 58(12), 1137-1143 (2015). https://doi.org/10.1097/dcr.0000000000000492

21. M. Galanopoulos, N. Evans, R. McFadyen, R. Naik, I. Drami, O. Ogunbiyi, T.V. Luong, J. Watkins, F. Laskaratos, D. Mandair, M. Caplin, C. Toumpanakis, Reassessment of risk factors associated with locoregional lymph nodal metastases in well-differentiated appendiceal neuroendocrine neoplasms. Neuroendocrinology 106, 79-79 (2018)

22. E.A. Woltering, Incidence of nodal positivity in appendiceal neuroendocrine tumors (NETs) and its effect on survival. Paper presented at the NANETS, Austin, Texas

23. F. Senel, H. Karaman, H. Demir, Neuroendocrine tumors detected in appendectomy specimens: ten-year single-center experience. Turk. J. Med. Sci. 48(1), 68-73 (2018). https://doi.org/10.3906/sa g-1709-37

24. A. Roggo, W.C. Wood, L.W. Ottinger, Carcinoid tumors of the appendix. Ann. Surg. 217(4), 385-390 (1993)
25. Z.M. Bamboat, D.L. Berger, Is right hemicolectomy for $2.0-\mathrm{cm}$ appendiceal carcinoids justified? Arch. Surg. 141(4), 349-352 (2006). https://doi.org/10.1001/archsurg.141.4.349. discussion 352

26. M. Buchholtz, J.M. Zuniga, F. Valdes, R. Fernandez, R. Neveu, C. Trujillo, G. Thonet, M. Carcamo, Carcinoid tumors. Retrospective review of 25 patients. Revista Chilena De Cirugia 62(5), 480-485 (2010)

27. J.R. Anderson, B.G. Wilson, Carcinoid tumours of the appendix. Br. J. Surg. 72(7), 545-546 (1985)

28. H. Wu, M. Chintagumpala, J. Hicks, J.G. Nuchtern, M.F. Okcu, R. Venkatramani, Neuroendocrine tumor of the appendix in children. J. Pediatr. Hematol./Oncol. 39(2), 97-102 (2017). https://doi.org/10.1097/mph.0000000000000598

29. N. Boxberger, A. Redlich, C. Böger, I. Leuschner, D. von Schweinitz, H. Dralle, P. Vorwerk, Neuroendocrine tumors of the appendix in children and adolescents. Pediatr. Blood Cancer 60 (1), 65-70 (2013). https://doi.org/10.1002/pbc.24267

30. G. De Lambert, H. Lardy, H. Martelli, D. Orbach, F. Gauthier, F. Guérin, Surgical management of neuroendocrine tumors of the appendix in children and adolescents: a retrospective french multicenter study of 114 cases. Pediatr. Blood Cancer 63(4), 598-603 (2016). https://doi.org/10.1002/pbc.25823

31. C.L. Moertel, L.H. Weiland, R.L. Telander, Carcinoid tumor of the appendix in the first two decades of life. J. Pediatr. Surg. 25 (10), 1073-1075 (1990)

32. S.E. Parkes, K.R. Muir, M. al Sheyyab, A.H. Cameron, J.R. Pincott, F. Raafat, J.R. Mann, Carcinoid tumours of the appendix in children 1957-1986: incidence, treatment and outcome. Br. J. Surg. 80(4), 502-504 (1993)

33. P.P. Parikh, E.A. Perez, H.L. Neville, A.R. Hogan, J.E. Sola, Nationwide overview of survival and management of appendiceal tumors in children. J. Pediatr. Surg. 53(6), 1175-1180 (2018). https://doi.org/10.1016/j.jpedsurg.2018.02.080

34. S.S. Kim, D.W. Kays, S.D. Larson, S. Islam, Appendiceal carcinoids in children-management and outcomes. J. Surg. Res. 192 (2), 250-253 (2014). https://doi.org/10.1016/j.jss.2014.06.031

35. K.S. Fernandez, J.H. Aldrink, M. Ranalli, F.B. Ruymann, D.A. Caniano, Carcinoid tumors in children and adolescents: risk for second malignancies. J. Pediatr. Hematol./Oncol. 37(2), 150-153 (2015). https://doi.org/10.1097/MPH.0000000000000280

36. A. Andersson, L. Bergdahl, Carcinoid tumors of the appendix in children. A report of 25 cases. Acta Chir. Scand. 143(3), 173-175 (1977)

37. C. Ricci, C. Ingaldi, L. Alberici, N. Brighi, D. Santini, C. Mosconi, V. Ambrosini, D. Campana, F. Minni, R. Casadei, Histopathological diagnosis of appendiceal neuroendocrine neoplasms: when to perform a right hemicolectomy? A systematic review and meta-analysis. Endocrine (2019). https://doi.org/10.1007/s12020019-01984-Z

38. J.A. Sterne, A.J. Sutton, J.P. Ioannidis, N. Terrin, D.R. Jones, J. Lau, J. Carpenter, G. Rucker, R.M. Harbord, C.H. Schmid, J. Tetzlaff, J.J. Deeks, J. Peters, P. Macaskill, G. Schwarzer, S. Duval, D.G. Altman, D. Moher, J.P. Higgins, Recommendations for examining and interpreting funnel plot asymmetry in metaanalyses of randomised controlled trials. BMJ 343, d4002 (2011). https://doi.org/10.1136/bmj.d4002 